\title{
One-sided ideals of the non-commutative Schwartz space
}

\author{
Krzysztof Piszczek ${ }^{1}$
}

Received: 16 July 2014 / Accepted: 9 March 2015 / Published online: 24 March 2015

(C) The Author(s) 2015. This article is published with open access at Springerlink.com

\begin{abstract}
We show that maximal one-sided ideals of the non-commutative Schwartz space are closed. We also characterize all closed one-sided ideals of this algebra. As a result, all maximal left ideals are fixed.
\end{abstract}

Keywords Fréchet space · (Fréchet) LMC-algebra · (one-sided) Ideal · Fixed ideal

Mathematics Subject Classification Primary 46H40 - 46H30 - 46K05;

Secondary $46 \mathrm{H} 20$

\section{Introduction}

The problem of characterizing ideals of a given topological algebra belongs to classical ones and there is vast literature devoted to it. The aim of this paper is to address this problem in the case of one particular lmc Fréchet *-algebra, the so-called noncommutative Schwartz space. This object has received reasonable attention recently, mostly due to Ciaś—see [3], Domański—see [7] and the author—see [21,22]. This paper is a continuation of the previous work. The motivation for the investigation of the non-commutative Schwartz space-which we will denote from now on by $\mathcal{S}$ comes from several directions. First, it has several, natural function (and sequence) space representations, for instance $\mathcal{S}$ is isomorphic (as a topological vector space)

Communicated by A. Constantin.

Krzysztof Piszczek

kpk@amu.edu.pl

1 Faculty of Mathematics and Computer Science, Adam Mickiewicz University in Poznań, ul. Umultowska 87, 61-614 Poznan, Poland 
to the space $C^{\infty}(M)$ of smooth functions on a compact smooth manifold $M$ or to the Schwartz space $\mathcal{S}(\mathbb{R})$ of test functions for tempered distributions (again as a tvs). This last isomorphism justifies also the name for $\mathcal{S}$ (the other name algebra of smooth operators - used e.g. by Ciaś - comes from the first-mentioned isomorphism). This links our object with the structure theory of Fréchet spaces, especially with questions concerning nuclearity or splitting of short exact sequences-see [17, Part IV]. This algebra appears also in the context of K-theory - see [4,20] or in the context of cyclic cohomology for crossed products—see [12,23]. Another interesting motivation comes from the theory of operator spaces and its locally convex analogues—see $[10,11]$. The non-commutative Schwartz space plays also a role in quantum mechanics, where it is called the space of physical states and its dual is the so-called space of observablessee [8] for details. This algebra shares also some nice features with $C^{*}$-algebras, e.g. it admits a Hölder functional calculus - see [3, Th. 5.2] and all positive functionals as well as all derivations are automatically continuous — see [21, Ths. 11, 13]. On the other hand it causes some technical difficulties, e.g. it has neither a bounded approximate identity—see [21, Prop. 2] nor is it a locally $\mathrm{C}^{*}$-algebra—see [13, Ths. 8.2, 8.3].

The paper is divided into four parts. The next section contains basic notation and terminology. The remaining two sections contain our main results. They are devoted only to one-sided ideals since $\mathcal{S}$ is topologically simple, i.e. there are no non-trivial closed two-sided ideals - see [21, Th. 4]. In the third Section we show that all maximal one-sided ideals of $\mathcal{S}$ are closed-see Theorem 5. In the last Section we characterize all closed one-sided ideals of $\mathcal{S}$ - see Theorem 7. As a consequence, we obtain that a left ideal of the non-commutative Schwartz space is maximal if and only if it is fixed-see Corollary 8.

For unexplained details we refer the reader to [17] in the case of the structure theory of Fréchet spaces and to [5] in the case of the 'algebraic-in-flavour' aspects of the paper.

The author is very indebted to Tomasz Kania for many valuable comments and discussions and to David Blecher for pointing out the reference [9].

\section{Notation and terminology}

Throughout the paper we denote $\mathbb{N}:=\{1,2,3, \ldots\}$ and $\mathbb{N}_{0}:=\mathbb{N} \cup\{0\}$. Recall that by

$$
s=\left\{\xi=\left(\xi_{j}\right)_{j \in \mathbb{N}} \subset \mathbb{C}^{\mathbb{N}}:|\xi|_{k}^{2}:=\sum_{j=1}^{+\infty}\left|\xi_{j}\right|^{2} j^{2 k}<+\infty \text { for all } k \in \mathbb{N}_{0}\right\}
$$

we denote the space of rapidly decreasing sequences. This space becomes Fréchet when endowed with the above defined sequence $\left(|\cdot|_{k}\right)_{k \in \mathbb{N}_{0}}$ of norms. The basis $\left(U_{k}\right)_{k \in \mathbb{N}_{0}}$ of zero neighbourhoods of $s$ is defined by $U_{k}:=\left\{\xi \in s:|\xi|_{k} \leq 1\right\}$. This space is topologically isomorphic to several spaces of functions, e.g. the space $C^{\infty}(M)$ of smooth functions on any smooth, compact manifold $M$ or the Schwartz space of rapidly decreasing functions $\mathcal{S}(\mathbb{R})$ — see [17, Example 29.5(2), (4)]. It is also present 
in the structure theory of (nuclear) Fréchet spaces as well as in the theory of splitting of short exact sequences of Fréchet spaces-see [17,25, Ch. 30]. The topological dual of $s$ is

$$
s^{\prime}=\left\{\eta=\left(\eta_{j}\right)_{j \in \mathbb{N}} \subset \mathbb{C}^{\mathbb{N}}:|\eta|_{k}^{\prime 2}:=\sum_{j=1}^{+\infty}\left|\eta_{j}\right|^{2} j^{-2 k}<+\infty \text { for some } k \in \mathbb{N}_{0}\right\} .
$$

If we now endow the space $\mathcal{S}:=L\left(s^{\prime}, s\right)$ of linear and continuous operators from the dual of $s$ into $s$ with the topology of uniform convergence on bounded sets then it becomes a Fréchet space with the sequence $\left(\|\cdot\|_{n}\right)_{n \in \mathbb{N}_{0}}$ of norms given by

$$
\|x\|_{n}:=\sup \left\{|x \xi|_{n}: \xi \in U_{n}^{\circ}\right\}
$$

where $U_{n}^{\circ}=\left\{\xi \in s^{\prime}: \quad \sum_{j}\left|\xi_{j}\right|^{2} j^{-2 n} \leq 1\right\}$. It is fairly easy to observe that the identity map $\iota: s \hookrightarrow s^{\prime}$ is a continuous embedding which allows us to endow the space $\mathcal{S}$ with the multiplication defined by

$$
x y:=x \circ \iota \circ y, \quad x, y \in \mathcal{S} \text {. }
$$

This multiplication is easily seen to be separately continuous therefore by [26, Th. $1.5]$ it is also jointly continuous. If we define the duality bracket between $s$ and $s^{\prime}$ by

$$
\langle\xi, \eta\rangle:=\sum_{j \in \mathbb{N}} \xi_{j} \bar{\eta}_{j}, \quad \xi \in s, \eta \in s^{\prime}
$$

then our algebra can also be given an involution map defined by

$$
\left\langle x^{*} \xi, \eta\right\rangle:=\langle\xi, x \eta\rangle, \quad x \in \mathcal{S}, \xi, \eta \in s^{\prime}
$$

With these operations $\mathcal{S}$ becomes a locally multiplicatively convex (lmc for short) Fréchet $*$-algebra and this will be the main object of the paper. It has several representations as the lmc Fréchet $*$-algebra—see e.g. [7, Th. 1.1]. Of particular interest for us will be the $*$-algebra isomorphism $\mathcal{S} \simeq \mathcal{K}^{\infty}$, where

$$
\mathcal{K}^{\infty}:=\left\{x=\left(x_{i, j}\right)_{i, j \in \mathbb{N}}:\|x\|_{n}^{2}:=\sum_{i, j=1}^{\infty}\left|x_{i j}\right|^{2}(i j)^{2 n}<+\infty \text { for all } n \in \mathbb{N}_{0}\right\}
$$

is the lmc Fréchet $*$-algebra endowed with the topology defined by the sequence of norms $\left(\|\cdot\|_{n}\right)_{n \in \mathbb{N}_{0}}$, multiplication of matrices and the 'star' operation being the matrix conjugate transpose. It turns out that we also have the isomorphism $\mathcal{S} \simeq s$-this time, however, as Fréchet spaces. Note that any reasonable multiplication (e.g. pointwise) on the sequence space $s$ makes it into a commutative algebra while $\mathcal{S}$ is highly noncommutative. Thus, as a Fréchet space, $\mathcal{S}$ can be thought of as the space of smooth functions or the Schwartz space of rapidly decreasing functions. On the other hand, if 
we endow $s$ with the pointwise multiplication and involution being the coordinatewise complex conjugation then we get a $*$-isomorphism (onto its range) $D: s \rightarrow \mathcal{S}$ sending a sequence $a=\left(a_{j}\right)$ into a diagonal operator $D_{a}:=\operatorname{diag}\left(a_{j}\right)_{j}$. The above-mentioned Fréchet space isomorphism $\mathcal{S} \simeq s$ justifies the name non-commutative Schwartz space which we will be using throughout the paper.

The isomorphism $\mathcal{S} \simeq \mathcal{K}^{\infty}$ shows that our algebra can be thought of as an algebra of matrices. Moreover the zeroth norm in $\mathcal{S}$ is just the $C^{*}$-algebra norm in $B\left(\ell_{2}\right)$ and so we obtain a continuous inclusion map $\mathcal{S} \hookrightarrow B\left(\ell_{2}\right)$. In fact we even have $\mathcal{S} \hookrightarrow K\left(\ell_{2}\right)$ since every element of $\mathcal{S}$ is a compact operator on $\ell_{2}$. The above inclusions have several consequences. Among them we point out that the non-commutative Schwartz space does not have a unit and so by $\mathcal{S}_{1}$ we denote its unitization. This unit is in fact the identity map on $\ell_{2}$ therefore we still have $\mathcal{S}_{1} \subset B\left(\ell_{2}\right)$. Furthermore by [7, Th. 2.3] an element $x \in \mathcal{S}_{1}$ is invertible in $\mathcal{S}_{1}$ if and only if it is invertible in $B\left(\ell_{2}\right)$. Recall now that by the natural inclusions $s \hookrightarrow \ell_{2}$ and $\ell_{2} \hookrightarrow s^{\prime}$ we have $\mathcal{S} B\left(\ell_{2}\right) \mathcal{S} \subset \mathcal{S}$. Therefore, if we denote by $\sigma_{A}(x)$ the spectrum of an element $x$ in the algebra $A$, by [2, Prop. A.2.8] (cf. [7, Cor. 2.5]) we have

$$
\sigma_{\mathcal{S}_{1}}(x)=\sigma_{B\left(\ell_{2}\right)}(x), \quad x \in \mathcal{S}_{1}
$$

This equality shows in particular that an element $x \in \mathcal{S}$ is positive in $\mathcal{S}$ if and only if it is positive as an operator on $\ell_{2}$. Consequently, the order structure, multiplication and the 'star' operation in $\mathcal{S}$ and $\mathcal{S}_{1}$ are inherited from the $\mathrm{C}^{*}$-algebra $B\left(\ell_{2}\right)$. Furthermore, the space of self-adjoint elements of $\mathcal{S}$, denoted by $\mathcal{S}_{s a}$ is a real Fréchet space and the set $\mathcal{S}_{+}$of positive elements is a convex cone in $\mathcal{S}$. Moreover by [7, Cor. 2.4] $\mathcal{S}_{1}$ is a Q-algebra and by [13, Ths. 8.2, 8.3] the topology of both $\mathcal{S}$ and $\mathcal{S}_{1}$ cannot be given by a sequence of $\mathrm{C}^{*}$-norms. It has also been shown recently in [3] that $\mathcal{S}$ as well as $\mathcal{S}_{1}$ admit a functional calculus for normal elements. Its description is a bit technical nevertheless we can work with the Hölder functions which vanish at 0 . In particular we can take roots of positive elements. To obtain this functional calculus one has to apply a purely Fréchet space property (called $(D N)$ ) which holds trivially in Banach spaces-see [3, Th. 5.2] for details.

\section{Closedness of maximal one-sided ideals}

In this Section we prove that all maximal one-sided ideals of the non-commutative Schwartz space are closed. Recall that one-sided ideals are in fact all ideals one can consider since by [21, Th. 4] $\mathcal{S}$ is topologically simple, i.e. there are no non-trivial two-sided ideals. We will show in fact a stronger property, namely that null sequences in $\mathcal{S}$ factor, i.e. for every sequence $\left(x_{n}\right)_{n \in \mathbb{N}} \subset \mathcal{S}$ tending to 0 there is a single element $a \in \mathcal{S}$ and another sequence $\left(y_{n}\right)_{n \in \mathbb{N}} \subset \mathcal{S}$ also tending to zero such that $x_{n}=a y_{n}$ for all $n \in \mathbb{N}$. This property may also be defined for algebra modules—see [5, Def. 2.6.11] - however we will not need this general setting here. It turns out that if null sequences in a Banach algebra factor then maximal right ideals are closed-see [14, Th. 3] (cf. also [5, Prop. 2.6.13]). However the proof of this result carries over onto 
Fréchet lmc algebras. If the algebra is involutive, this applies also to maximal left ideals. We state this result explicitly.

Theorem 1 If null sequences in a Fréchet lmc *-algebra factor then all maximal one-sided ideals are closed.

In order to prove that null sequences in $\mathcal{S}$ factor we cannot follow the Banach-algebra proof of Varopoulos, who proved in [24] that if a Banach algebra admits a bounded approximate identity then null sequences in this algebra factor. Instead we will rely on [1] and [9]. Our proof will be divided into several steps. We start by defining $\alpha$ additive ordered Fréchet spaces (we restrict ourselves to the Fréchet-space setting but this definition can mutatis mutandis be extended onto arbitrary lcs). By an ordered locally convex space we mean a real lcs partially ordered by a closed convex cone of positive elements. Our definition is slightly different from the one used e.g. by Asimov in the setting of Banach spaces_-see [1, p. 118]. While the dual to a Banach space is still a Banach space (with the dual order if the initial space was ordered), this is no longer true for Fréchet spaces (these are called (DF)-spaces which are not metrizable in general). Therefore we think such a slight change is reasonable. Of course for Banach ordered spaces (which are by definition also Fréchet spaces) we get two different notions of $\alpha$-additiveness. The relation between these notions for an arbitrary ordered Banach space $X$ is that it is $\alpha$-additive in our sense if and only if the dual ordered Banach space $X^{\prime}$ (with the dual order) is $\alpha$-additive in the sense used by Asimov.

Definition 2 Let $\alpha \geqslant 0$. An ordered Fréchet space $X$ is $\alpha$-additive if for any $p \in \mathbb{N}$ there exists $q \in \mathbb{N}$ such that for any $n \in \mathbb{N}$ and an arbitrary choice of $n$ positive functionals $\phi_{1}, \ldots, \phi_{n} \in X^{\prime}$ we have that

$$
\sum_{k=1}^{n}\left\|\phi_{k}\right\|_{q}^{*} \leq \alpha\left\|\sum_{k=1}^{n} \phi_{k}\right\|_{p}^{*} .
$$

Proposition $3 \mathcal{S}_{s a}$ is $\frac{\pi^{2}}{6}$-additive.

Proof We will be using in the proof the representation $\mathcal{S} \simeq \mathcal{K}^{\infty}$ with the $\ell_{\infty}$-norms of the matrices that is

$$
\|x\|_{k, \infty}:=\sup \left\{\left|x_{i j}\right|(i j)^{k}: i, j \in \mathbb{N}\right\} .
$$

Consequently, the dual norms in $\mathcal{S}^{\prime}$ are given by the formulae

$$
\|\phi\|_{k, 1}^{*}:=\sum_{i, j=1}^{+\infty}\left|\phi_{i j}\right|(i j)^{-k} \quad\left(\phi \in \mathcal{S}^{\prime}\right) .
$$

Take a single positive functional $\phi$. Positivity then implies—see [18, Ex. 3.2(i), p. 39] or the proof of [21, Lemma 5]-that

$$
\left|\phi_{i j}\right|^{2} \leq \phi_{i i} \phi_{j j} \quad \text { for all } i, j \in \mathbb{N} \text {. }
$$


By Cauchy-Schwarz this leads to

$$
\begin{aligned}
\|\phi\|_{k, 1}^{*} & =\sum_{i, j=1}^{+\infty}\left|\phi_{i j}\right|(i j)^{-k} \leq\left(\sum_{i, j=1}^{+\infty}\left|\phi_{i j}\right|^{2}(i j)^{-2(k-1)}\right)^{\frac{1}{2}}\left(\sum_{i, j=1}^{+\infty}(i j)^{-2}\right)^{\frac{1}{2}} \\
& \leq \frac{\pi^{2}}{6}\left(\sum_{i, j=1}^{+\infty} \phi_{i i} \phi_{j j}(i j)^{-2(k-1)}\right)^{\frac{1}{2}}=\frac{\pi^{2}}{6} \sum_{j=1}^{+\infty} \phi_{j j} j^{-2(k-1)}
\end{aligned}
$$

For arbitrary positive functionals $\phi^{1}, \ldots, \phi^{n}$ this gives

$$
\sum_{m=1}^{n}\left\|\phi^{m}\right\|_{k}^{*} \leq \frac{\pi^{2}}{6} \sum_{j=1}^{+\infty}\left(\sum_{m=1}^{n} \phi_{j j}^{m}\right) j^{-2(k-1)} \leq \frac{\pi^{2}}{6}\left\|\sum_{m=1}^{n} \phi^{m}\right\|_{k-1,1}^{*} .
$$

We have thus shown that for any $k \in \mathbb{N}_{0}, n \in \mathbb{N}$ and arbitrary choice of positive functionals $\phi^{1}, \ldots \phi^{n} \in \mathcal{S}^{\prime}$

$$
\sum_{m=1}^{n}\left\|\phi^{m}\right\|_{k+1,1}^{*} \leq \frac{\pi^{2}}{6}\left\|\sum_{m=1}^{n} \phi^{m}\right\|_{k, 1}^{*}
$$

This proves that $\mathcal{S}_{s a}$ is $\frac{\pi^{2}}{6}$-additive.

The next step is to show that $\mathcal{S}_{s a}$ is quasi-directed. An ordered locally convex space is (see [9]) quasi-directed if for any neighbourhood $U$ of zero there is another neighbourhood $V$ of zero such that every finite subset of $V$ is majorized by some element of $U$. We will now prove that the ordered self-adjoint part of the non-commutative Schwartz space is quasi-directed. The idea of proof comes from the proof of [1, Th. 1.1]. However at some point we will have to modify it in order to adapt it to the Fréchet-space setting.

Proposition $4 \mathcal{S}_{\text {sa }}$ is quasi-directed.

Proof Let $\alpha=\frac{\pi^{2}}{6}$ and fix $n \in \mathbb{N}$. Following Asimov we denote by $\mathcal{S}_{\mathbf{s a}}^{(\mathbf{n})}$ the $n$-fold Cartesian product of $\mathcal{S}_{s a}$ with itself. The topology in this Fréchet space is given by the basis $\left(\mathbf{U}_{\mathbf{k}}^{(\mathbf{n})}\right)_{k \in \mathbb{N}_{0}}$ of zero neighbourhoods, where

$$
\mathbf{U}_{\mathbf{k}}^{(\mathbf{n})}=\left\{\mathbf{x}^{(\mathbf{n})}=\left(x_{1}, \ldots, x_{n}\right) \in \mathcal{S}_{\mathbf{s a}}^{(\mathbf{n})}:\left\|\mathbf{x}^{(\mathbf{n})}\right\|_{k}:=\max \left\{\left\|x_{j}\right\|_{k}: j=1, \ldots, n\right\} \leq 1\right\} .
$$

With the closed positive cone $\mathbf{P}^{(\mathbf{n})}:=\left\{\mathbf{p}^{(\mathbf{n})}: p_{j} \geqslant 0, j=1, \ldots, n\right\} \mathcal{S}_{\mathbf{s a}}^{(\mathbf{n})}$ becomes an ordered Fréchet space. We also define $\mathbf{D}_{\mathbf{k}}^{(\mathbf{n})}:=\left\{\mathbf{x}^{(\mathbf{n})}=(x, \ldots, x):\|x\|_{k} \leq \alpha\right\}$ to be the diagonal in $\mathcal{S}_{\text {sa }}^{(\mathbf{n})}$ and

$$
\mathbf{A}_{\mathbf{k}}^{(\mathbf{n})}:=\mathbf{D}_{\mathbf{k}}^{(\mathbf{n})}-\mathbf{P}^{(\mathbf{n})}=\left\{\left(x-p_{1}, \ldots, x-p_{n}\right):\|x\|_{k} \leq \alpha, p_{j} \geqslant 0\right\} .
$$

Following exactly the proof of [1, Th. 1.1] we obtain that 


$$
\mathbf{U}_{\mathbf{k}+\mathbf{1}}^{(\mathbf{n})} \subset \overline{\mathbf{A}_{\mathbf{k}}^{(\mathbf{n})}} \quad\left(k \in \mathbb{N}_{0}\right)
$$

Now we modify the proof of Asimov. Let $\mathbf{x}^{(\mathbf{n})} \in \mathbf{U}_{\mathbf{k}+\mathbf{1}}^{(\mathbf{n})}$. By (2) there is $\mathbf{z}_{\mathbf{1}}^{(\mathbf{n})}-\mathbf{p}_{\mathbf{1}}^{(\mathbf{n})} \in$ $\mathbf{A}_{\mathbf{k}}^{(\mathbf{n})}$ such that

$$
\left\|\mathbf{x}^{(\mathbf{n})}-\left(\mathbf{z}_{\mathbf{1}}^{(\mathbf{n})}-\mathbf{p}_{\mathbf{1}}^{(\mathbf{n})}\right)\right\|_{k+2}<\frac{1}{2}, \quad\left\|\mathbf{z}_{\mathbf{1}}^{(\mathbf{n})}\right\|_{k} \leq \alpha, \quad \mathbf{p}_{\mathbf{1}}^{(\mathbf{n})} \geqslant 0
$$

If $\mathbf{y}_{\mathbf{1}}^{(\mathbf{n})}:=\mathbf{x}^{(\mathbf{n})}-\left(\mathbf{z}_{\mathbf{1}}^{(\mathbf{n})}-\mathbf{p}_{\mathbf{1}}^{(\mathbf{n})}\right)$ then $\mathbf{x}^{(\mathbf{n})}=\mathbf{y}_{\mathbf{1}}^{(\mathbf{n})}+\mathbf{z}_{\mathbf{1}}^{(\mathbf{n})}-\mathbf{p}_{\mathbf{1}}^{(\mathbf{n})} \leq \mathbf{y}_{\mathbf{1}}^{(\mathbf{n})}+\mathbf{z}_{\mathbf{1}}^{(\mathbf{n})}$ and $\mathbf{y}_{\mathbf{1}}^{(\mathbf{n})} \in \frac{1}{2} \overline{\mathbf{A}_{\mathbf{k}+\mathbf{1}}^{(\mathbf{n})}}$. Therefore there is $\mathbf{z}_{\mathbf{2}}^{(\mathbf{n})}-\mathbf{p}_{\mathbf{2}}^{(\mathbf{n})} \in \frac{1}{2} \mathbf{A}_{\mathbf{k}+\mathbf{1}}^{(\mathbf{n})}$ such that

$$
\left\|\mathbf{y}_{\mathbf{1}}^{(\mathbf{n})}-\left(\mathbf{z}_{\mathbf{2}}^{(\mathbf{n})}-\mathbf{p}_{\mathbf{2}}^{(\mathbf{n})}\right)\right\|_{k+3}<\frac{1}{2^{2}}, \quad\left\|\mathbf{z}_{\mathbf{2}}^{(\mathbf{n})}\right\|_{k+1} \leq \frac{\alpha}{2}, \quad \mathbf{p}_{\mathbf{2}}^{(\mathbf{n})} \geqslant 0 .
$$

If $\mathbf{y}_{\mathbf{2}}^{(\mathbf{n})}:=\mathbf{y}_{\mathbf{1}}^{(\mathbf{n})}-\left(\mathbf{z}_{\mathbf{2}}^{(\mathbf{n})}-\mathbf{p}_{\mathbf{2}}^{(\mathbf{n})}\right)$ then $\mathbf{x}^{(\mathbf{n})} \leq \mathbf{z}_{\mathbf{1}}^{(\mathbf{n})}+\mathbf{z}_{\mathbf{2}}^{(\mathbf{n})}+\mathbf{y}_{\mathbf{2}}^{(\mathbf{n})}$ and $\mathbf{y}_{\mathbf{2}}^{(\mathbf{n})} \in \frac{1}{2^{2}} \mathbf{A}_{\mathbf{k}+\mathbf{2}}^{(\mathbf{n})}$. Proceeding recursively we will find sequences $\left(\mathbf{y}_{\mathbf{m}}^{(\mathbf{n})}\right)_{m \in \mathbb{N}},\left(\mathbf{z}_{\mathbf{m}}^{(\mathbf{n})}\right)_{m \in \mathbb{N}} \subset \mathcal{S}_{\text {sa }}$ such that $\left\|\mathbf{y}_{\mathbf{m}}^{(\mathbf{n})}\right\|_{k+1+m} \leq \frac{1}{2^{m}}, \quad \mathbf{z}_{\mathbf{m}}^{(\mathbf{n})} \in \frac{1}{2^{m-1}} \mathbf{D}_{\mathbf{k}-\mathbf{1}+\mathbf{m}}^{(\mathbf{n})}, \quad \mathbf{x}^{(\mathbf{n})} \leq \mathbf{z}_{\mathbf{1}}^{(\mathbf{n})}+\cdots+\mathbf{z}_{\mathbf{m}}^{(\mathbf{n})}+\mathbf{y}_{\mathbf{m}}^{(\mathbf{n})} \quad(m \in \mathbb{N})$

It is now easy to show that $\mathbf{y}_{\mathbf{m}}^{(\mathbf{n})} \rightarrow 0$ when $m$ tends to infinity and the series $\sum_{m=1}^{+\infty} \mathbf{z}_{\mathbf{m}}^{(\mathbf{n})}$ converges absolutely. Therefore there is $\mathbf{z}^{(\mathbf{n})}:=\sum_{m=1}^{+\infty} \mathbf{z}_{\mathbf{m}}^{(\mathbf{n})}$ and $\mathbf{x}^{(\mathbf{n})} \leq \mathbf{z}^{(\mathbf{n})}$. Moreover

$$
\left\|\mathbf{z}^{(\mathbf{n})}\right\|_{k} \leq \sum_{m=1}^{+\infty}\left\|\mathbf{z}_{\mathbf{m}}^{(\mathbf{n})}\right\|_{k-1+m} \leq 2 \alpha
$$

The above argument was independent of the choice of $n \in \mathbb{N}$ which means that for any $k \in \mathbb{N}_{0}, n \in \mathbb{N}$ and for arbitrary self-adjoint $x_{1}, \ldots, x_{n} \in U_{k+1}$ there is $z \in 2 \alpha U_{k}$ such that $x_{j} \leq z$ for all $j=1, \ldots, n$. Since $\left(U_{k}\right)_{k}$ is a basis of zero neighbourhoods this implies that $\mathcal{S}_{s a}$ is quasi-directed.

Now we are ready to prove the following result.

Theorem 5 Null sequences in the non-commutative Schwartz space factor. Consequently, all maximal one-sided ideals of $\mathcal{S}$ are closed.

Proof Let $\left(x_{n}\right)_{n \in \mathbb{N}}$ be a null sequence in $\mathcal{S}$. By [21, Lemmata 8 and 12] we have

$$
x_{n}=\left(x_{n} x_{n}^{*}\right)^{\frac{1}{4}} y_{n}, \quad\left\|y_{n}\right\|_{k, \infty} \leq \sqrt{2}\left\|x_{n}\right\|_{4 k, \infty} \quad(n \in \mathbb{N})
$$

where $\|a\|_{k, \infty}=\sup \left\{\left|a_{i j}\right|(i j)^{k}: i, j \in \mathbb{N}\right\}$ for $a \in \mathcal{S}$. This implies that the sequences $\left(y_{n}\right)_{n \in \mathbb{N}} \in \mathcal{S}$ and $\left(z_{n}\right)_{n \in \mathbb{N}} \in \mathcal{S}_{+}, z_{n}:=\left(x_{n} x_{n}^{*}\right)^{\frac{1}{4}}$ both tend to zero. By Proposition 4 and [9, Th. 1] there is a positive element $z \in \mathcal{S}$ which majorizes the sequence $\left(z_{n}\right)_{n}$, 
i.e. $z_{n} \leq z$ for all $n \in \mathbb{N}$. Now we apply [21, Lemma 12] to the pairs $\left(z, z_{n}\right)$ and we obtain a decomposition

$$
z_{n}^{\frac{1}{2}}=z^{\frac{1}{4}} w_{n}, \quad\left\|w_{n}\right\|_{k, \infty} \leq \sqrt{2} \max \left\{\left\|z^{\frac{1}{4}}\right\|_{2 k, \infty},\left\|z_{n}^{\frac{1}{2}}\right\|_{4 k, \infty}^{\frac{1}{2}}\right\} \quad(n \in \mathbb{N}) .
$$

Consequently,

$$
x_{n}=z_{n} y_{n}=z^{\frac{1}{4}}\left(w_{n} z^{\frac{1}{4}} w_{n} y_{n}\right):=z^{\frac{1}{4}} t_{n} \quad(n \in \mathbb{N}) .
$$

By (3) the sequence $\left(w_{n} z^{\frac{1}{4}} w_{n}\right)_{n \in \mathbb{N}}$ is bounded and $y_{n} \rightarrow 0$ therefore the sequence $\left(t_{n}\right)_{n \in \mathbb{N}}$ is a null sequence and the proof is complete.

Remark The $\ell_{\infty}$-norms of the sequence $\left(w_{n}\right)_{n \in \mathbb{N}}$ in (3) can in fact be controlled in a more convenient way. More precisely, since the squre root is an operator monotone function on $[0,+\infty)$-see [19, Prop. 1.3.8], we can combine this fact with [21, Cor. 6, Lemma 8] in order to get $\left\|w_{n}\right\|_{k, \infty} \leq \sqrt{2}\left\|z^{\frac{1}{2}}\right\|_{4 k, \infty}^{\frac{1}{2}}$ for all $n \in \mathbb{N}$.

\section{Description of closed one-sided ideals}

The description we are going to provide is analogous to the characterization of closed one-sided ideals of the algebra of compact (or nuclear) operators on a Hilbert space - see [16, Th. (III. 2.5)]. The proof of this result relies strongly on the fact that every closed subspace in a Hilbert space is complemented which is not the case for the Schwartz space. Therefore we choose to adapt the proof of [15, Prop. 7.3]. The general idea is the same however in several points we have to appeal to the fact that we are dealing precisely with the Schwartz space instead of just a Banach space with the approximation property (which is the case in [15]).

Recall for convenience that

$$
s=\left\{\xi=\left(\xi_{j}\right)_{j \in \mathbb{N}} \subset \mathbb{C}^{\mathbb{N}}:|\xi|_{k}^{2}:=\sum_{j=1}^{+\infty}\left|\xi_{j}\right|^{2} j^{2 k}<+\infty \text { for all } k \in \mathbb{N}_{0}\right\} .
$$

In particular the zeroth norm $\|\cdot\|_{0}$ is just the $\ell_{2}$-norm of a rapidly decreasing sequence and we can compute the inner product $\langle\cdot, \cdot\rangle$ of two vectors in $s$. In this case this inner product is in fact given by the duality (1). The definition below which we start with goes back to Grønbæk's paper. Let $F$ be a closed subspace in $s$ and let $I$ be a closed left ideal of $\mathcal{S}$. We define

$$
\begin{aligned}
& \Phi(F):=\varlimsup i n\{\xi \otimes \eta: \quad \xi \in s, \eta \in F\}, \\
& \Psi(I):=\{\eta \in s: \quad \exists \xi \neq 0: \quad \xi \otimes \eta \in I\} .
\end{aligned}
$$

Remark It can be easily observed that $\Phi(F)$ is a closed left ideal of $\mathcal{S}$. Moreover, $\Psi(I)$ is a closed subspace of $s$. Indeed, take any non-zero $\zeta \in s$ and let $\eta \in \Psi(I)$. By 
definition there is some non-zero element $\xi \in s$ such that $\xi \otimes \eta \in I$. Since $I$ is a left ideal we get

$$
\zeta \otimes \eta=\left(\zeta \otimes \frac{1}{\|\xi\|_{0}^{2}} \xi\right)(\xi \otimes \eta) \in I
$$

Therefore

$$
\Psi(I)=\{\eta \in s \mid \forall \xi \in s: \xi \otimes \eta \in I\}
$$

Let now $\eta_{n} \rightarrow \eta,\left(\eta_{n}\right)_{n \in \mathbb{N}} \subset \Psi(I)$. For arbitrary $\xi \in s$ we have

$$
\xi \otimes \eta=\lim _{n} \xi \otimes \eta_{n} \in I
$$

since $I$ is closed. Consequently, $\eta \in \Psi(I)$.

Proposition 6 Let $F$ be a closed subspace in $s$ and let I be a closed left ideal of $\mathcal{S}$. Then

(i) $I=\Phi(\Psi(I))$,

(ii) $F=\Psi(\Phi(F))$.

Proof (i) The inclusion ' $\supset$ ' is clear. To show the other one choose $x \in I$ and for every $n \in \mathbb{N}$ define $u_{n}:=\left(\begin{array}{cc}I_{n} & 0 \\ 0 & 0\end{array}\right)$, where $I_{n}$ is the $n \times n$ identity map. Then for all $n \in \mathbb{N}$ we have $u_{n} x \in I$ and $\operatorname{dim} \operatorname{im} u_{n} x<+\infty$. We may suppose that

$$
u_{n} x=\sum_{j=1}^{m_{n}} \xi_{j} \otimes \eta_{j}, \quad \xi_{j}, \eta_{j} \in s, j=1, \ldots, m_{n}
$$

and the vectors $\left(\xi_{j}\right)_{j}$ are linearly independent. By the Gram-Schmidt orthogonalization we obtain orthogonal vectors $e_{1}, \ldots, e_{m_{n}} \in \ell_{2}$ satisfying

$$
\left\langle\xi_{m_{n}}, e_{m_{n}}\right\rangle \neq 0 \text { and }\left\langle\xi_{j}, e_{m_{n}}\right\rangle=0 \quad\left(j=1, \ldots, m_{n}-1\right) .
$$

But $e_{j}$ 's are linear combinations of $\xi_{j}$ 's therefore $\left(e_{j}\right)_{j=1}^{m_{n}} \subset s$. By (5) this leads to

$$
\xi_{m_{n}} \otimes \eta_{m_{n}}=\frac{1}{\left\langle\xi_{m_{n}}, e_{m_{n}}\right\rangle}\left(\xi_{m_{n}} \otimes e_{m_{n}}\right) u_{n} x \in I .
$$

Reenumerating the indices in (4) if necessary,

$$
\xi_{j} \otimes \eta_{j} \in I \quad\left(j=1, \ldots, m_{n}\right)
$$

Therefore

$$
\eta_{j} \in \Psi(I) \quad\left(j=1, \ldots, m_{n}\right) .
$$


Consequently, $u_{n} x \in \Phi(\Psi(I))$ for all $n \in \mathbb{N}$. By [21, Prop. 2] $\left(u_{n}\right)_{n \in \mathbb{N}}$ is a sequential approximate identity therefore $x \in \Phi(\Psi(I))$ and the desired equality follows.

(ii) This time the inclusion ' $C$ ' is clear. Let now $\eta \in \Psi(\Phi(F))$ and take $\xi \neq 0$ such that $\xi \otimes \eta \in \Phi(F)$. By definition

$\xi \otimes \eta=\lim _{n \rightarrow+\infty} x_{n}, \quad x_{n}=\sum_{j=1}^{m_{n}} \xi_{j}^{n} \otimes \eta_{j}^{n}, \quad \xi \in s, \eta_{j}^{n} \in F \quad\left(n \in \mathbb{N}, j=1, \ldots, m_{n}\right)$.

Let $\zeta:=\frac{1}{\|\xi\|_{0}^{2}}$ and define $\eta_{n}:=\sum_{j=1}^{m_{n}}\left\langle\xi_{j}^{n}, \zeta\right\rangle \eta_{j}^{n}$. Then each $\eta_{n} \in F$ and $(\xi \otimes \zeta) x_{n}=$ $\xi \otimes \eta_{n}$. This implies that

$$
\xi \otimes \eta=\lim _{n \rightarrow+\infty} \xi \otimes \eta_{n}
$$

Consequently, for every $k \in \mathbb{N}_{0}$ we have

$$
\left\|\eta-\eta_{n}\right\|_{k}=\frac{1}{\|\xi\|_{k}}\left\|\xi \otimes \eta-\xi \otimes \eta_{n}\right\|_{k} \rightarrow 0
$$

Therefore $\eta \in F$ and the proof is thereby complete.

Theorem 7 Let I be a closed one-sided ideal of the non-commutative Schwartz space.

(i) If I is a left ideal then there is a closed subspace $E \subset s^{\prime}$ such that

$$
I=\left\{x \in \mathcal{S}:\left.x\right|_{E} \equiv 0\right\}
$$

(ii) If I is a right ideal then there is a closed subspace $E \subset s^{\prime}$ such that

$$
I=\left\{x \in \mathcal{S}: \overline{\operatorname{im} x}=E^{\perp}\right\}
$$

Proof (i) Let $I$ be a closed left ideal of $\mathcal{S}$ and define $E:=\cap_{\eta \in \Psi(I)} \operatorname{ker} \eta \subset s^{\prime}$ and $J:=\left\{x \in \mathcal{S}:\left.x\right|_{E} \equiv 0\right\}$. It is clear that $\Phi(\Psi(I)) \subset J$ and so by Proposition 6(i) we get $I \subset J$. On the other hand $J=\Phi(\Psi(J))$ since it is a closed left ideal of $\mathcal{S}$. Suppose now $\eta \in \Psi(J)$. Then $\Psi(J)^{\perp} \subset(\mathbb{C} \cdot \eta)^{\perp}$ and by the Bipolar Theorem $[17$, Th. 22.13] $\mathbb{C} \cdot \eta \subset \Psi(I)$ (recall these are closed subspaces). Consequently, $\Psi(J) \subset \Psi(I)$ and $\Phi(\Psi(J)) \subset \Phi(\Psi(I))$. Thus again by Proposition 6(i) $J \subset I$ and the equality follows.

(ii) If $I$ is a closed right ideal then $I^{*}:=\left\{x^{*}: x \in I\right\}$ is a closed left ideal. By (i) there is $E \subset s^{\prime}$ such that $I^{*}=\left\{x^{*}:\left.x^{*}\right|_{E} \equiv 0\right\}$. Using now the duality (1) it is easy to obtain the desired conclusion.

For maximal left ideals the above characterization may be rephrased in the language of fixed ideals. Following [6] a fixed ideal is a left ideal $I$ of the form

$$
I=I_{\xi}:=\{x \in \mathcal{S}: x(\xi)=0\} \quad\left(\xi \in s^{\prime} \backslash\{0\}\right) .
$$


Corollary 8 A left ideal of the non-commutative Schwartz space is maximal if and only if it is fixed.

Proof If $I$ is a maximal left ideal then by Theorem 5 it is closed. Therefore by Theorem 7(i) $I=\left\{x \in \mathcal{S}:\left.x\right|_{E} \equiv 0\right\}$ for some closed subspace $E \subset s^{\prime}$. Maximality of $I$ gives now $\operatorname{dim} E=1$. On the other hand every fixed ideal is maximal. Indeed, let $I_{\xi}$ be a fixed ideal and take $y \in \mathcal{S}$ with $0 \neq y(\xi) \in s$. This means that $\left\langle y(\xi), e_{j}\right\rangle$ for some coordinate functional $e_{j}: s \rightarrow \mathbb{C}, e_{j}(\eta):=\eta_{j}$. But such a functional is easily seen to be a rapidly decreasing sequence therefore there exists $\mu \in s$ such that $\langle y(\xi), \mu\rangle=1$. For any $n \in \mathbb{N}$ denote $\xi^{n}:=\left(\xi_{1}, \ldots, \xi_{n}, 0,0, \ldots\right)$. Then $\left(u_{n}-\left(\xi^{n} \otimes \mu\right) y\right)(\xi)=0$. Thus $u_{n}-\left(\xi^{n} \otimes \mu\right) y \in I \xi$ and, consequently,

$$
u_{n}=u_{n}-\left(\xi^{n} \otimes \mu\right) y+\left(\xi^{n} \otimes \mu\right) y \in\left\langle I_{\xi} \cup\{y\}\right\rangle \quad(n \in \mathbb{N}) .
$$

Since $\left(u_{n}\right)_{n \in \mathbb{N}} \subset \mathcal{S}$ is an approximate identity, we arrive at $\mathcal{S}=\left\langle I_{\xi} \cup\{y\}\right\rangle$ and the proof is complete.

Acknowledgments The research of the author has been supported in the years 2014-2017 by the National Center of Science, Poland, Grant no. DEC-2013/10/A/ST1/00091.

Open Access This article is distributed under the terms of the Creative Commons Attribution License which permits any use, distribution, and reproduction in any medium, provided the original author(s) and the source are credited.

\section{References}

1. Asimov, L.: Directed Banach spaces of affine functions. Trans. Am. Math. Soc. 143, 117-132 (1969)

2. Bost, J.-B.: Principe d'Oka, $K$-théorie et systèmes dynamiques non commutatifs. Invent. Math. 101(2), 261-333 (1990)

3. Ciaś, T.: On the algebra of smooth operators. Stud. Math. 218(2), 145-166 (2013)

4. Cuntz, J.: Cyclic Theory and the Bivariant Chern-Connes Character. In: Noncommutative geometry, vol. 1831. Lecture Notes in Math. Springer, Berlin, pp. 73-135 (2004)

5. Dales, H.G.: Banach Algebras and Automatic Continuity, vol. 24. London Mathematical Society Monographs. New Series. The Clarendon Press, Oxford University Press, New York, Oxford Science Publications 2000

6. Dales, H.G., Kania, T., Kochanek, T., Koszmider, P., Laustsen, N.J.: Maximal left ideals of the Banach algebra of bounded operators on a Banach space. Stud. Math. 218(3), 245-286 (2013)

7. Domański, P.: Algebra of smooth operators. http://main3.amu.edu.pl/domanski/salgebra1.pdf (unpublished manuscript)

8. Dubin, D.A., Hennings, M.A.: Quantum Mechanics, Algebras and Distributions, vol. 238. Pitman Research Notes in Mathematics Series. Longman Scientific \& Technical. Harlow; copublished in the United States with Wiley, New York, 1990

9. Duhoux, M., Ng, K.F.: Decomposition of precompact operators in ordered locally convex spaces. J. Lond. Math. Soc. (2) 13(3), 387-392 (1976)

10. Effros, E.G., Webster, C.: Operator analogues of locally convex spaces. In: Operator algebras and applications (Samos, 1996), vol. 495. NATO Adv. Sci. Inst. Ser. C Math. Phys. Sci. Kluwer Acad. Publ., Dordrecht, pp. 163-207 (1997)

11. Effros, E.G., Winkler, S.: Matrix convexity: operator analogues of the bipolar and Hahn-Banach theorems. J. Funct. Anal. 144(1), 117-152 (1997)

12. Elliott, G.A., Natsume, T., Nest, R.: Cyclic cohomology for one-parameter smooth crossed products. Acta Math. 160(3-4), 285-305 (1988)

13. Fragoulopoulou, M.: Topological Algebras with Involution. North-Holland Mathematics Studies, vol. 200. Elsevier Science B.V, Amsterda (2005) 
14. Green, M.D.: Maximal one-sided ideals in Banach algebras. Math. Proc. Camb. Philos. Soc. 80(1), 109-111 (1976)

15. Grønbæk, N.: Morita equivalence for Banach algebras. J. Pure Appl. Algebra 99(2), 183-219 (1995)

16. Helemskii, A.Y.: Banach and Locally Convex Algebras. Oxford Science Publications. The Clarendon Press, Oxford University Press, New York (1993). Translated from the Russian by A. West

17. Meise, R., Vogt, D.: Introduction to Functional Analysis. Oxford Graduate Texts in Mathematics, vol. 2. The Clarendon Press, Oxford University Press, New York (1997). Translated from the German by M. S. Ramanujan and revised by the authors

18. Paulsen, V.: Completely Bounded maps and Operator Algebras. Cambridge Studies in Advanced Mathematics, vol. 78. Cambridge University Press, Cambridge (2002)

19. Pedersen, G.K.: $C^{*}$-algebras and their Automorphism Groups. London Mathematical Society Monographs, vol. 14. Academic Press, Inc. [Harcourt Brace Jovanovich, Publishers], London-New York (1979)

20. Phillips, N.C.: K-theory for Fréchet algebras. Int. J. Math. 2(1), 77-129 (1991)

21. Piszczek, K.: Automatic continuity and amenability in the noncommutative Schwartz space. In: Workshop on Functional Analysis Valencia 2013, Adam Mickiewicz University in Poznań, Valencia, Spain 3-6 June 2013

22. Piszczek, K.: A Jordan-like decomposition in the noncommutative Schwartz space. Bull. Aust. Math. Soc. 91(2), 322-330

23. Schweitzer, L.B.: Spectral invariance of dense subalgebras of operator algebras. Int. J. Math. 4(2), 289-317 (1993)

24. Varopoulos, N.Th: Sur les formes positives d'une algèbre de Banach. C. R. Acad. Sci. Paris 258, 2465-2467 (1964)

25. Vogt, D.: On the functors $\operatorname{Ext}^{1}(E, F)$ for Fréchet spaces. Stud. Math. 85(2), 163-197 (1987)

26. Żelazko, W.: Selected topics in topological algebras. Matematisk Institut, Aarhus Universitet, Aarhus, Lectures 1969/1970. Lecture Notes Series, No. 31 (1971) 